\title{
Placement of C2 laminar screws using three-dimensional fluoroscopy-based image guidance by Eric W. Nottmeier and Andrew B. Foy
}

\author{
J. J. Verlaan
}

Accepted: 6 December 2007/ Published online: 3 January 2008

(C) The Author(s) 2007

In their work colleagues Nottmeier and Foy present a retrospective series of eight patients treated with posterior $\mathrm{C} 1-\mathrm{C} 2$ fusion by laminar screws using three-dimensional fluoroscopy-based image guidance. They conclude from the results of their work that: "In this study, it has been shown that C2 laminar screws can be safely and accurately placed using 3D fluoroscopy-based image guidance. Advantages of this technology over traditional techniques include real-time 3D computerized feedback to the surgeon during placement, as well as the ability to size the screw to the patient's anatomy using intraoperative computerized planning."

In the last decade, technology has advanced to the point where regular computer workstations are able to calculate in real-time the transformation matrices needed to determine the position of a camera-tracked object (in a surgical setting, typically a frame with fiducials) in relation to a camera-tracked reference base. By attaching the reference base to a patient and (surgical) instruments to cameratracked objects, the workstation can calculate the respective positions/orientations and display their virtual interactions. When three-dimensional data sets representing the patient's anatomy from modalities including CT/ $\mathrm{MRI} / 3 \mathrm{D}$ rotational X-ray imaging, are manipulated so that virtual and real reference bases are exactly aligned, surgical instruments and/or implants can be displayed on anatomical structures not visible for the surgeon

This reviewer's comment refers to the article http://dx.doi.org/10.1007/s00586-007-0557-x

J. J. Verlaan $(\bowtie)$

Department of Orthopaedics, University Medical Center Utrecht,

Heidelberglaan 100, 3584CX Utrecht, The Netherlands

e-mail: jj.verlaan@wxs.nl intraoperatively. This is, in a nutshell, current "computerassisted surgery" or "image-guided surgery". Assuming that transformation matrix calculations are mathematical processes carried out with high fidelity and great reproducibility, imperfections in navigational accuracy must have other sources. Calibration errors in 3D fluoroscopybased systems have, for example, been shown to account for approximately $1 \mathrm{~mm}$ accuracy in a phantom model [1]. In a more realistic cadaveric model, the accuracy of navigation deteriorated to approximately $2.5 \mathrm{~mm}$, mainly due to bending of instruments and/or references during manipulation [2]. In clinical practice more inaccuracies can be introduced by occasional blocking of the camera field of view; inadvertently touching/hitting references and, especially, a nonrigid connection between the patient reference base and the actual surgical site. In the present study, the patient reference base was attached to a head clamp, which was, in turn, connected via pins to the patient's skull while surgery was performed at the levels $\mathrm{C} 1$ and $\mathrm{C} 2$. This experimental setup represents quite a long chain of connections from the reference base to the surgical site with considerable opportunity for movement between the two extremes. This is without taking into account additional movements due to, for example, patient ventilation and direct manipulation of $\mathrm{C} 1$ and $\mathrm{C} 2$ by surgical instruments. Considering that the $\mathrm{C} 0-\mathrm{C} 1-\mathrm{C} 2$ complex is highly mobile, it came as a small surprise for this reviewer to find such accurate screw placement in the current study. As can be found in the article, the investigators did verify the imageguided technique ("After accuracy of navigation was confirmed by the surgeon ..."). The interesting question that follows automatically is: "How?" Checking accuracy by probing the patient reference base with a navigated instrument and finding a perfectly matched position/orientation on the computer display does nothing for the 
flexibility/movement errors that may be present at the more distant surgical site. Most often, surgeons verify accuracy by a direct intraoperative view of the surgical site to see whether, for example, the proposed drill trajectory or planned screw length is in accordance to their own experience from conventional surgeries before moving on with navigation. This leads to the strange paradox that is really bugging contemporary image-guided procedures: the employment of a technique to enhance surgical accuracy requiring the surgeon's intraoperative verification of its accuracy. The reluctance of surgeons to show complete faith in image guidance seems justified considering the errors demonstrated in accuracy studies. Using computer navigation without proper anatomical and surgical knowledge would be irresponsible. Armed with proper knowledge, however, navigation becomes largely superfluous since surgeons trust their surgical experience/skills and the direct intraoperative view over computer-generated images anytime, and adjust their actions accordingly. Which surgeon would happily volunteer when asked to perform a percutaneous procedure having to trust solely on information provided by any image-guided technique commercially available today?
Until now, no large-scale long-term and properly controlled study has been published in the field of computer-assisted surgery that has convincingly showed any actual patient benefit when using image guidance. Lacking a control group and using a navigation technique susceptible to the aforementioned accuracy problems, the present study may, unfortunately, not be able to change this status.

Open Access This article is distributed under the terms of the Creative Commons Attribution Noncommercial License which permits any noncommercial use, distribution, and reproduction in any medium, provided the original author(s) and source are credited.

\section{References}

1. van de Kraats EB, van Walsum T, Kendrick L et al (2006) Accuracy evaluation of direct navigation with an isocentric 3D rotational X-ray system. Med.Image Anal 10:113-124

2. van de Kraats EB, van Walsum T, Verlaan JJ et al. (2006) Threedimensional rotational X-ray navigation for needle guidance in percutaneous vertebroplasty: an accuracy study. Spine 31:13591364 